\title{
Diffusion-weighted magnetic resonance imaging for assessment after neoadjuvant chemotherapy in breast cancer, based on morphological concepts
}

\author{
YORIKO MURATA $^{1}$, KEI KUBOTA ${ }^{1}$, NORIHIKO HAMADA ${ }^{1}$, KANA MIYATAKE ${ }^{1}$, MICHIKO TADOKORO ${ }^{1}$, \\ KIMIKO NAKATANI ${ }^{1}$, HIRONOBU UE ${ }^{1}$, KAZUHIRO TSUZUKI ${ }^{1}$, AKIHITO NISHIOKA ${ }^{1}$, \\ MITSUKO IGUCHI $^{2}$, HIRONOBU MAEDA ${ }^{3}$ and YASUHIRO OGAWA ${ }^{1}$
}

Departments of ${ }^{1}$ Radiology, ${ }^{2}$ Pathology, and ${ }^{3}$ Surgery, Kochi Medical School, Kochi 783-8505, Japan

Received June 24, 2009; Accepted January 8, 2010

DOI: 10.3892/ol_00000052

\begin{abstract}
The study aimed to evaluate the utility of diffusionweighted imaging (DWI) and to assess the response of breast cancer patients to neoadjuvant chemotherapy (NAC), based on morphological concepts. This retrospective study included 35 breast cancer patients (36 lesions) who had conventional magnetic resonance imaging (MRI), with DWI acquired before and after NAC. The morphological pattern of delayed enhancement on MRI before NAC was classified into two types: focal mass (FM), and multiple masses and/or non-mass like (MM/NM), based on Breast Imaging Reporting and Data System (BI-RADS). Of the 36 tumors, 26 were classified as $\mathrm{FM}$ and 10 as MM/NM. Tumors were clearly visualized on the initial DWI although one case of suspected MM/NM was not observed on DWI following NAC. A correlation was found between changes in the apparent diffusion coefficient and response rates to NAC in FM tumors $(r=0.608, p<0.001)$, but not in MM/NM tumors $(r=0.141, p=0.717)$. There was agreement between MRI findings after NAC and pathological findings in 30 of the 36 tumors (83.3\%). Thus, we concluded that DWI is potentially useful in assessing the response to NAC for breast cancer for tumors diagnosed as FM on the initial conventional MRI.
\end{abstract}

\section{Introduction}

Neoadjuvant chemotherapy (NAC) is currently recognized as an important component of breast cancer treatment for patients who i) are candidates for breast-conserving surgery, ii) have advanced-stage disease but are not candidates and iii) refuse surgery. Among the various tools for monitoring the response to NAC for breast cancer, contrast-enhanced magnetic reso-

Correspondence to: Dr Yoriko Murata, Department of Radiology, Kochi Medical School, Kohasu, Nankoku, Kochi 785-8505, Japan E-mail: muratay@kochi-u.ac.jp

Key words: breast cancer, magnetic resonance imaging, neoadjuvant chemotherapy nance imaging (MRI) is considered to be superior to any other imaging modality due to its high spatial resolution (1-6).

Developments have enabled diffusion-weighted imaging (DWI) to be used more widely in the study of breast cancer (7-11). Previous reports described the use of DWI in the differentiation of malignant from benign breast cancer (7,9-12). Apparent diffusion coefficient (ADC) obtained in DWI is reported to be useful for predicting early tumor response to NAC (12-14). In contrast to conventional MRI, DWI does not require the use of contrast-enhancing material and has good temporal resolution.

Breast cancer can be classified into several patterns based on morphology (5,15-17). In addition, the pattern of response to NAC depends largely on the initial morphology $(15,16)$. Focal or solitary nodular type tumors usually shrink centrically, while non-focal or unlocalized tumors shrink in a multicentric manner. Therefore, the response to NAC does not always correspond to shrinkage over the extent of the tumor. Optimal response in the solid nodular pattern is shrinkage of the tumor, which may have the potential to scatter small components within the range of the initial tumor bed in the multiple nodular and/or unlocalized dendritic patterns (16). Breast cancer treatment therefore requires different approaches for the two morphological patterns $(15,16)$. According to previous reports, focal or solitary nodular type tumors may correspond to a focal mass, while non-focal or multiple nodular and/or unlocalized dendritic type tumors may correspond to multiple masses and/or non-mass-like tumor, based on the Breast Imaging Reporting and Data System (BI-RADS) (18).

We hypothesized that the effectiveness of imaging parameters to gauge response to NAC in breast cancer may not be uniform across all subclasses of tumors. Therefore, the aim was to assess the efficacy of DWI of the breast to evaluate the response to NAC in breast cancer patients based on tumor morphology on MRI.

\section{Materials and methods}

Patients. Between April 2007 and September 2008, breast MRI was performed at our hospital on 200 patients with breast cancer. This retrospective study comprised 35 breast 
cancer patients (36 lesions) who underwent breast MRI before and after NAC. The time interval between MRI and the last course of chemotherapy ranged from 3 to 15 days (mean 7). Patient age ranged from 26 to 69 years (mean 54). According to the tumor-node-metastases (TNM) classification, the clinical stages of the tumors were IIA $(n=17)$, IIB $(n=8)$, IIIA $(n=7)$, IIIB $(n=2)$ and IIIC $(n=2)$. Patients were treated with anthracycline-based (EC) chemotherapy, and received four courses of epirubicine at $100 \mathrm{mg} / \mathrm{m}^{2}$ and cyclophosphamide at $600 \mathrm{mg} / \mathrm{m}^{2}$. Written informed consent was obtained from all subjects.

Magnetic resonance imaging. MRI was performed using a 1.5 T system (Signa HDx; General Electric Healthcare, USA). The patients were examined in the prone position using a breast coil. Dynamic MRI using a three-dimensional fast spoiled gradient-echo sequence (VIBRANT, volume imaging for breast imaging; TR $5.0 \mathrm{~ms}$; TE $2.7 \mathrm{~ms}$; flip angle $10^{\circ}$; FOV 28x $28 \mathrm{~cm}$; matrix 512x256; slice thickness $3 \mathrm{~mm}$; space $0 \mathrm{~mm}$; NEX 1) was obtained before and 8 times (every $30 \mathrm{sec}$ ) after a bolus injection of $0.1 \mathrm{mmol}$ gadoliniumdiethylenetriamine pentaacetic acid (Gd-DTPA)/kg by automatic injector at a rate of $3 \mathrm{ml} / \mathrm{sec}$, followed by a $50 \mathrm{ml}$ saline flush. Bilateral transverse diffusion-weighted images with b-values of 0 and $1,500 \mathrm{~s} / \mathrm{mm}^{2}$ were acquired before the administration of contrast material (TR $5000 \mathrm{~ms}$; TE $68.0 \mathrm{~ms}$; flip angle $90^{\circ}$; FOV 36x36 cm; matrix 160x160; slice thickness $3 \mathrm{~mm}$; space $0 \mathrm{~mm}$; NEX 4).

MRI data analysis. Delayed enhanced images were classified as focal mass (FM) or multiple masses and/or non-mass (MM/ $\mathrm{NM}$ ), based on BI-RADS (18). ADC values were calculated according to the formula: $A D C=[\operatorname{In} S(h) / S(l)] /(b(h)-b(l))$, where In is the natural $\log$, and $\mathrm{S}(\mathrm{h})$ and $\mathrm{S}(\mathrm{l})$ are the signal intensities in each region of interest (ROI), placed on sections that correspond to two different $b$ factors $\left(b=1500,0 \mathrm{~s} / \mathrm{mm}^{2}\right]$. In obtaining ADC values of the lesions, ROIs were placed carefully within the largest section of the tumor on DWI. The change in ADC following NAC was calculated as: (ADC after NAC - ADC before NAC)/(ADC before NAC) x100. The largest possible ROI was used according to the size and morphology of each tumor. The same radiologist calculated the ADCs of the tumors before and after NAC. Tumor sizes were measured on delayed enhanced MRI using the image with maximum tumor diameter and signal intensity of the tumor relative to the signal intensity of surrounding breast tissue. Tumor size was calculated as the bi-axial diameter product using maximum and orthogonal diameter on the maximum dimension of each tumor. Tumor response to NAC was calculated as: (tumor size before NAC - tumor size after NAC)/(tumor size before NAC) x100.

Dynamic enhancement was evaluated by visual assessment of the resulting curve, and delayed-enhancement patterns were classified as washout, plateau or persistent, based on BI-RADS (18). A ROI was placed manually on the portion of the lesion with the most rapid enhancement or the portion with the most suspicious washout curve.

Tumor morphologies and enhancement patterns were evaluated independently by one radiologist, and DWI by another. The two radiologists had $>10$ years of experience in the field of breast MRI.
Table I. Clinical manifestation and imaging findings of the cases examined.

\begin{tabular}{|c|c|c|}
\hline Characteristic & $\mathrm{FM}$ & $\mathrm{MM} / \mathrm{NM}$ \\
\hline \multicolumn{3}{|l|}{ Age (years) } \\
\hline Median & 55 & 56 \\
\hline Range & $26-69$ & $44-68$ \\
\hline \multicolumn{3}{|l|}{ TNM } \\
\hline IIA & 17 & 0 \\
\hline IIB & 6 & 2 \\
\hline IIIA & 1 & 6 \\
\hline IIIB & 1 & 1 \\
\hline IIIC & 1 & 1 \\
\hline \multicolumn{3}{|c|}{$\mathrm{ADC}\left(\mathrm{x} 10^{-3} \mathrm{~mm}^{2} / \mathrm{s}\right)$} \\
\hline \multicolumn{3}{|l|}{ Before NAC } \\
\hline Average & 0.833 & 0.815 \\
\hline $\mathrm{SD}$ & 0.153 & 0.146 \\
\hline \multicolumn{3}{|l|}{ After NAC } \\
\hline Average & 0.963 & 1.137 \\
\hline $\mathrm{SD}$ & 0.216 & 0.245 \\
\hline \multicolumn{3}{|c|}{ Change in ADC (\%) } \\
\hline Average & 17 & 50.1 \\
\hline $\mathrm{SD}$ & 23.6 & 58.9 \\
\hline \multicolumn{3}{|c|}{ Size in MRI $\left(\mathrm{mm}^{2}\right)$} \\
\hline \multicolumn{3}{|l|}{ Before NAC } \\
\hline Average & 531.1 & 2,088 \\
\hline $\mathrm{SD}$ & 524.4 & 1,583 \\
\hline \multicolumn{3}{|l|}{ After NAC } \\
\hline Average & 288.4 & $1,376.2$ \\
\hline SD & 277.2 & 1,048 \\
\hline \multicolumn{3}{|c|}{ Response rate $(\%)$} \\
\hline Average & 44.5 & 34.2 \\
\hline $\mathrm{SD}$ & 27.7 & 17.6 \\
\hline
\end{tabular}

FM, focal mass; MM/NM, multiple masses and/or non-mass; ADC, apparent diffusion coefficient; TNM, tumor-node-metastases classification and SD, standard deviation.

Surgical approach and histological evaluation. In breastconserving surgery, tumors were generally resected with a $2-\mathrm{cm}$ surgical margin. Breast specimens were evaluated by routine pathologic examination. The specimens were processed by serial gross sectioning at $\sim 1-\mathrm{cm}$ intervals. The closest margin was evaluated histologically. In cases where it was difficult to distinguish the extent of residual tumor on MRI after NAC, a skin marker (alfacalcidol capsule of $0.5 \mu \mathrm{g}$ for osteoporosis) was placed on the skin immediately before scanning in the supine position. Skin markers were helpful in evaluating the correlation between MRI measurement and histological size.

Statistical analysis. Pearson's correlation test was used to measure the linear association between ADC and clinical tumor measurements. A non-parametric test was used to assess significance in measuring the differences between 
A

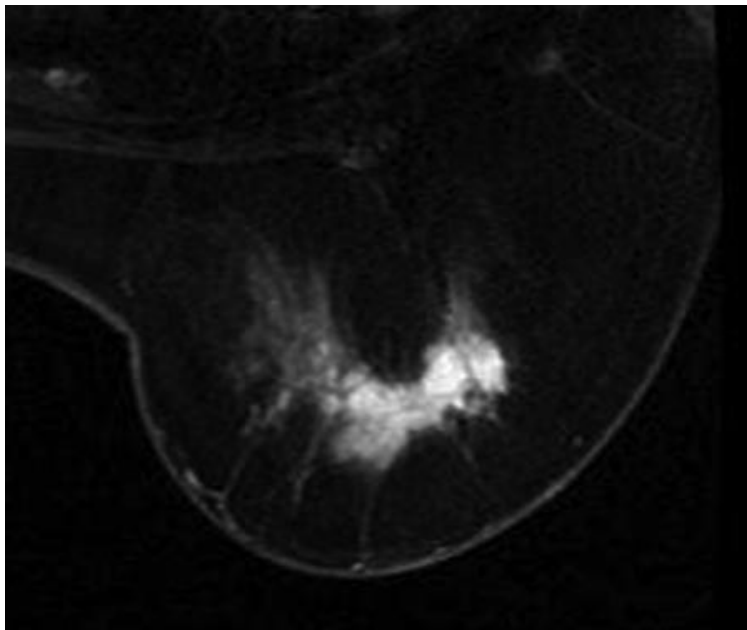

B

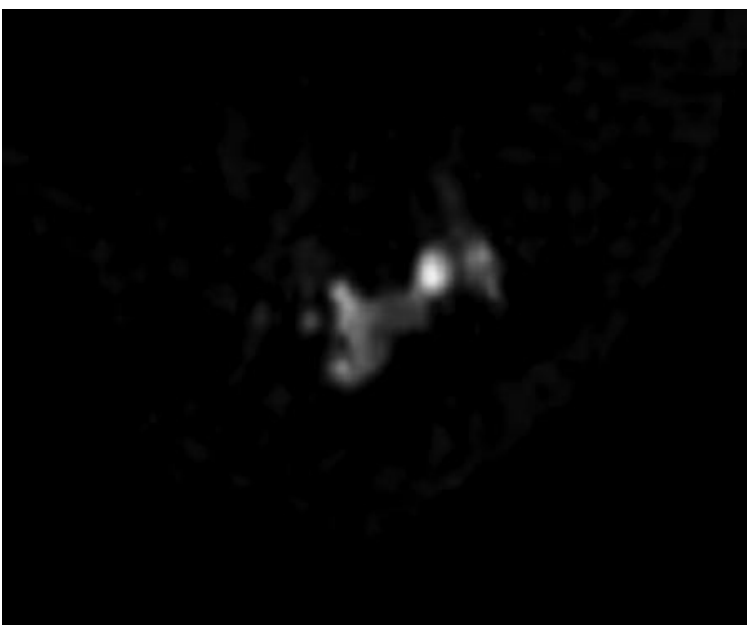

C

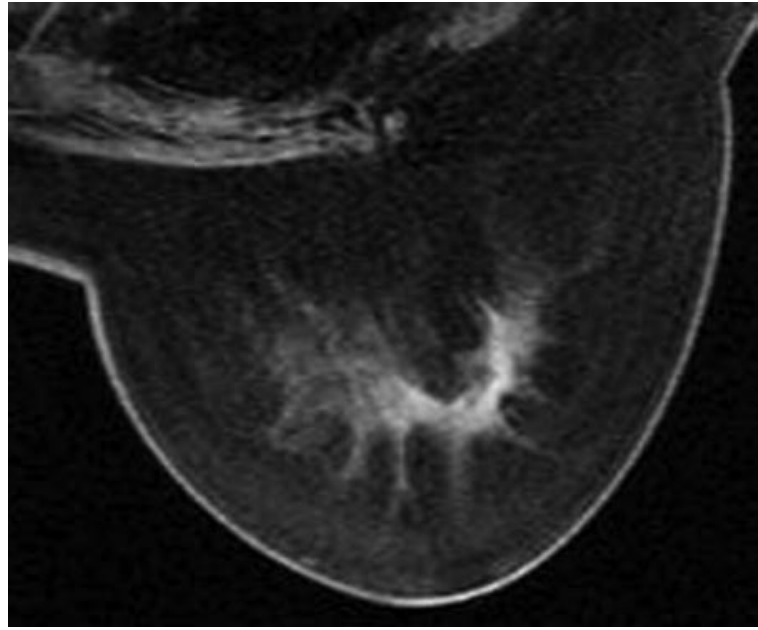

D

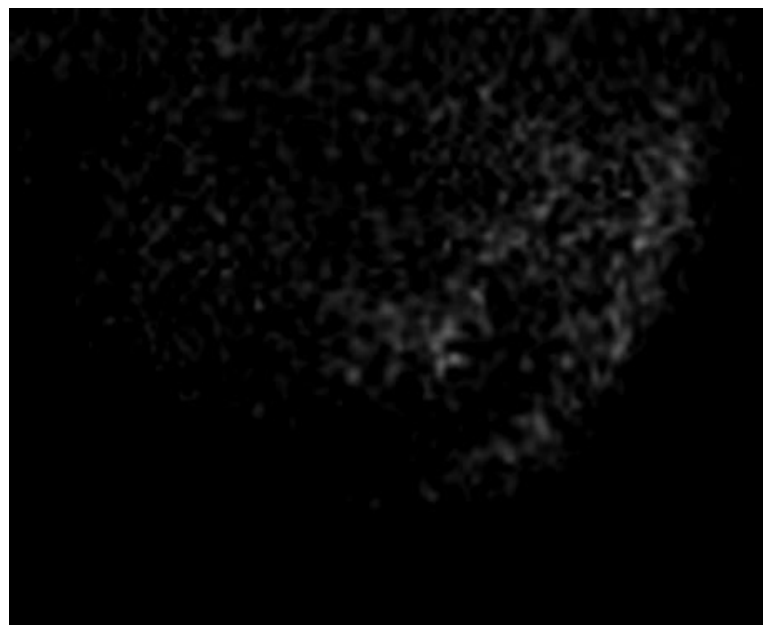

Figure 1. (A) A 50-year-old woman with invasive ductal carcinoma of the right breast. Three-dimensional contrast-enhanced fat-suppressed MRI of the breast acquired before NAC reveals an area of non-mass-like enhancement. (B) Before NAC, the tumor is clearly detected as an area of high intensity on the diffusion-weighted image. (C) Three-dimensional contrast-enhanced fat-suppressed MRI of the breast acquired after NAC reveals an area of decreased nonmass-like enhancement. (D) After NAC, the residual tumor is not detected on the diffusion-weighted image. The change in ADC cannot be calculated and response rate is $63.3 \%$. The extent of the residual tumor on the enhanced MRI corresponded approximately to the surgical findings by mastectomy.

groups. The Mann-Whitney U test was employed to measure the differences between the mean ADC of responders and non-responders before and after NAC. $\mathrm{P}<0.01$ was assumed to indicate a statistically significant difference.

\section{Results}

Patient characteristics and MRI findings are summarized in Table I. Breast-conserving surgery was performed in 29 patients (30 lesions), total glandectomy in 3 and total mastectomy in 3. The histological types of carcinoma included invasive ductal $(\mathrm{n}=34)$, mucinous $(\mathrm{n}=1)$ and invasive lobular carcinoma $(n=1)$. Assessment of the tumor response by MRI was in agreement with the pathological findings in $83 \%$ of cases (FM 23/26; MM/NM, 7/10). Imaging assessment overestimated the degree of response in 1 case $(\mathrm{FM})$ with extensive intraductal components and in 3 cases (MM/NM) with scattered invasive foci surrounding the lesions. The degree of response was underestimated in 2 cases (FM) of pathological complete response (pCR). In cases of MN/NM with a high ADC response, despite a low MRI response, extensive scattering of the residual tumor was identified histologically.
The tumors were well visualized on delayed enhancement MRI before and after NAC. Before NAC, the average maximum tumor size on MRI was $531.1 \mathrm{~mm}^{2}$ for $\mathrm{FM}$ $(\mathrm{SD}=524.4 ; \mathrm{n}=26)$ and $2,088 \mathrm{~mm}^{2}$ for $\mathrm{MM} / \mathrm{NM}(\mathrm{SD}=1,583$; $\mathrm{n}=10$ ). Of the FM tumors, all except 2 had the appearance of a focal mass after NAC, while 9 of the $10 \mathrm{MM} / \mathrm{NM}$ tumors had multiple masses or a non-mass-like appearance after NAC. After NAC, the average maximum tumor size was $288.4 \mathrm{~mm}^{2}$ for FM (SD=277.2) and 1,376.2 $\mathrm{mm}^{2}$ for MM/NM (SD=1048). The average response rate according to tumor size on MRI was $44.5 \%$ for FM (SD=27.7) and 34.2\% for MM/NM (SD=17.6).

The tumors were clearly visualized on DWI before NAC; all except one were visualized after NAC. The average ADC value before $\mathrm{NAC}$ was $0.833 \times 10^{-3} \mathrm{~mm}^{2} / \mathrm{s}$ for $\mathrm{FM}(\mathrm{SD}=0.153)$ and $0.815 \times 10^{-3} \mathrm{~mm}^{2} / \mathrm{s}$ for $\mathrm{MM} / \mathrm{NM}(\mathrm{SD}=0.146)$. The average ADC value after NAC was $0.963 \times 10^{-3} \mathrm{~mm}^{2} / \mathrm{s}$ for $\mathrm{FM}$ $(\mathrm{SD}=0.216)$ and $1.137 \times 10^{-3} \mathrm{~mm}^{2} / \mathrm{s}$ for $\mathrm{MM} / \mathrm{NM}(\mathrm{SD}=0.245)$. The average change in ADC was $17 \%$ for $\mathrm{FM}(\mathrm{SD}=23.6)$ and $50.1 \%$ for $\mathrm{MM} / \mathrm{NM}(\mathrm{SD}=58.9)$. An $\mathrm{MM} / \mathrm{NM}$ tumor that was not observed on DWI after NAC was clearly visualized on conventional MRI as having a $20 \%$ response rate, which was confirmed by the pathological findings. 
A

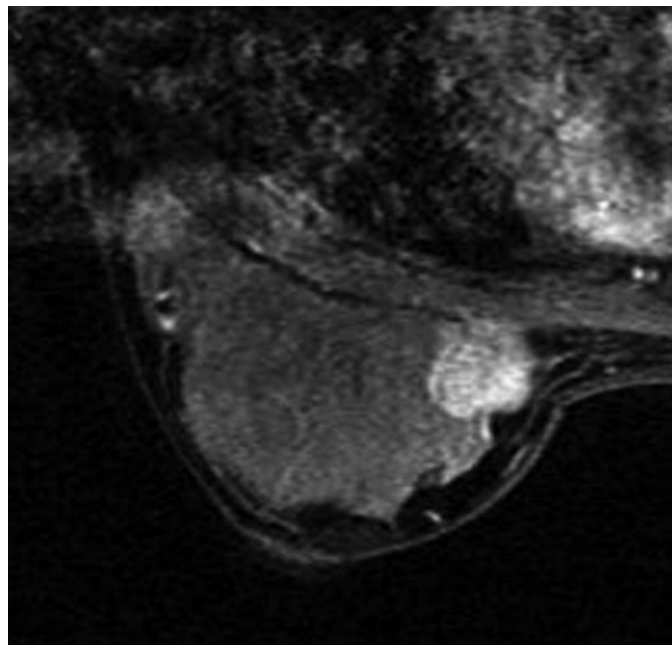

B

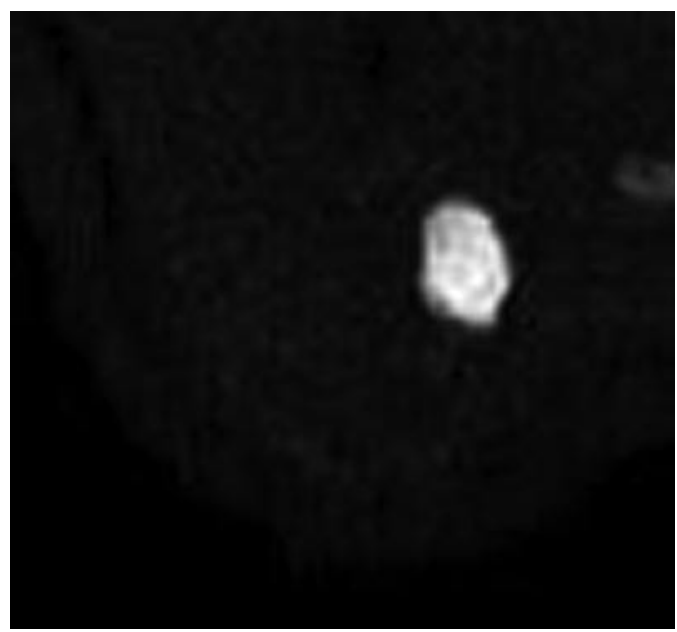

C

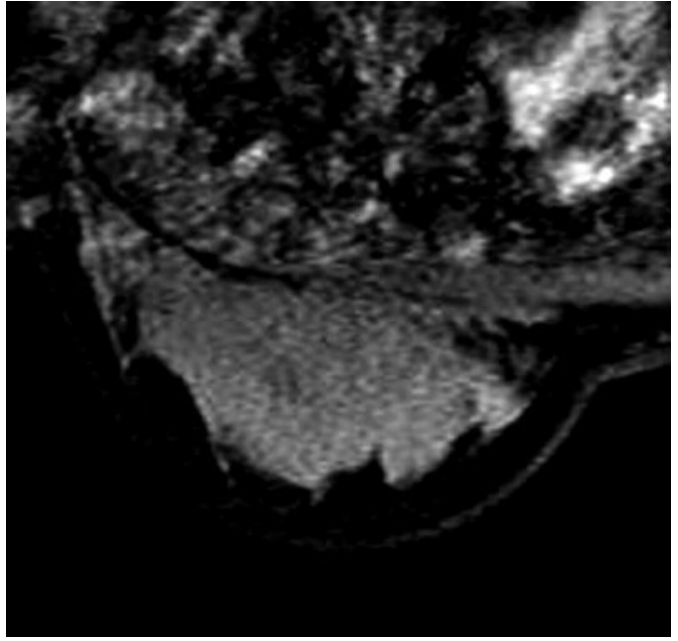

D

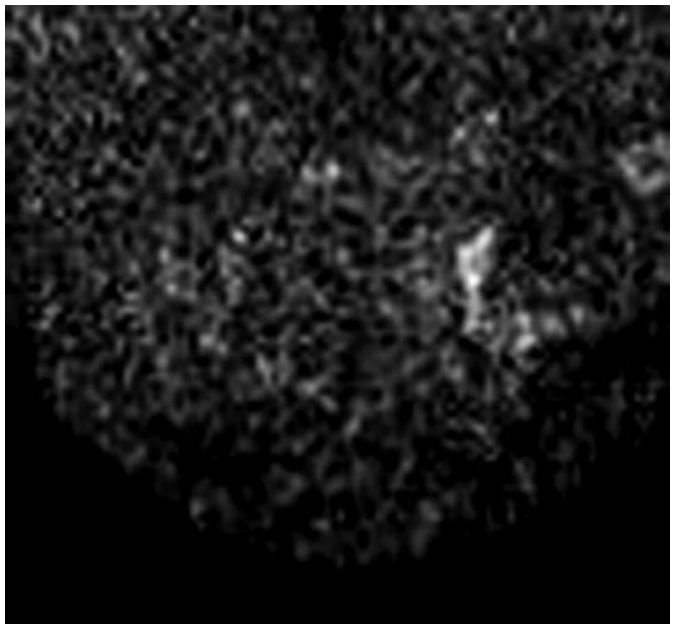

Figure 2. (A) A 26-year-old woman with invasive ductal carcinoma of the right breast. Three-dimensional contrast-enhanced fat-suppressed MRI of the breast acquired before NAC reveals a localized enhancing mass. (B) Before NAC, the tumor is clearly detected as an area of high intensity on the diffusion-weighted image. (C) Three-dimensional contrast-enhanced fat-suppressed MRI of the breast acquired after NAC reveals a significantly decreased residual mass. (D) After NAC, the tumor is detected as an area of high intensity on the diffusion-weighted image, smaller in size compared to that on the initial MRI. The change in ADC is $75.2 \%$ and the response rate is $91 \%$. The extent of the residual tumor on the enhanced MRI corresponded approximately to the surgical findings by mastectomy.

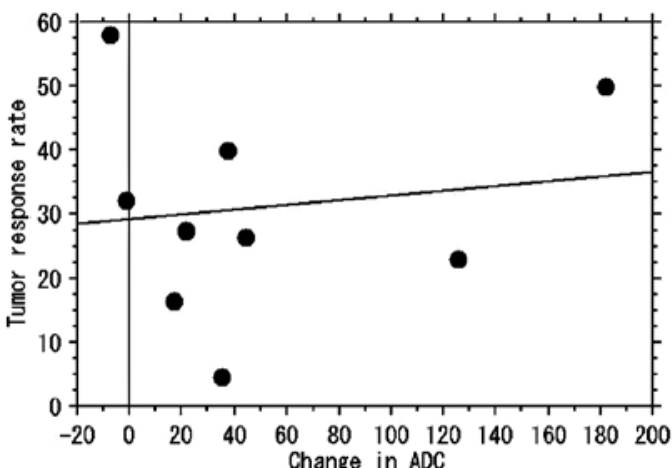

Figure 3. Correlation between changes in ADC and the response rate in MRI by NAC in MM/NM tumors.

For the MM/NM tumors, no correlation was found between change in $\mathrm{ADC}$ and the tumor reduction rate $(\mathrm{r}=0.141, \mathrm{p}=0.717)$ (Figs. 1 and 3). For 26 localized tumors, a positive correlation was found between change in ADC and the tumor reduction rate $(r=0.608, p<0.001)$ (Figs. 2 and 4). Assessment of the

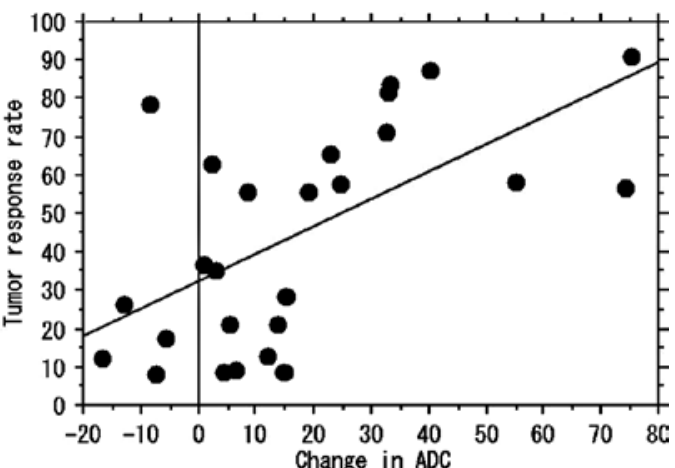

Figure 4. Correlation between changes in ADC and the response rate in MRI by NAC in FM tumors.

delayed enhancement patterns from dynamic MRI before NAC for the 36 tumors revealed washout in 5 and plateau in 31 tumors. Persistent delayed enhancement pattern was displayed in 12 cases of $\mathrm{FM}$ and 8 cases of MM/NM; the remaining 16 (FM, 14; MM/NM, 2) tumors demonstrated washout or plateau 
after NAC. For FM, change in the delayed enhancement pattern was significantly correlated with the response rate $(\mathrm{p}=0.008)$ and change in $\mathrm{ADC}(\mathrm{p}=0.004)$. However, no such correlations were found for MM/NM ( $\mathrm{p}=0.431, \mathrm{p}=0.449)$.

\section{Discussion}

MRI-based assessment commonly under- or overestimates the extent of residual breast cancer following NAC $(6,19)$. However, among the various imaging modalities, dynamicenhanced MRI is recognized as the most sensitive and has the highest spatial resolution. The use of morphological concepts can reduce the discrepancy between MRI assessment and pathological findings with regard to the extent of residual tumors $(15,16)$. The six under- or overestimated cases of MRI in the present study are considered to be an exception, particularly in the FM cases with extensive intraductal components and in the three $\mathrm{MM} / \mathrm{NM}$ cases with scattered invasive foci surrounding the tumors. Multiple nodular and/or unlocalized dendritic tumors usually demonstrate dendritic or multicentric shrinking and remain extensively in the original tumor beds $(15,16)$. In the two cases of pCR, the conventional MRI and DWI findings may represent so-called pseudotumors corresponding to xanthomatous change (20).

The clinical usefulness of DWI has already been established in patients with cerebral infarction in the acute stage (21-23). Tumors are depicted as regions of high signal intensity, since their ADC values are lower than those for normal tissue. The applications of DWI have expanded to various organs (24-27). In DWI, ADC reflects the biological character of the tissue. Thus, ADC values in the breast are useful in differentiating malignant from benign tumors, or in predicting response to chemotherapy (7,9-14).

Despite its excellent contrast resolution, DWI is vastly inferior to conventional studies, since its spatial resolution is reduced by susceptibility and chemical shift artifacts (28). Furthermore, cell density is reportedly related to $\operatorname{ADC}(7,29)$. Previous studies reported that DWI was not able to depict a small compartment of focus of ductal carcinoma in situ neighboring a main tumor, comedo-type ductal carcinoma in situ containing notable bleeding or necrosis $(9,11)$. In the present study, one case was not visualized on DWI after NAC. However, enhanced MRI showed an obvious residual tumor that was able to be proven pathologically. In two other cases with a discrepancy between the change in ADC and response to NAC on MRI, extensive scattering of the residual tumor was observed in the original tumor bed, although the central regions of tumors and tumor cell density were not analyzed histologically in these cases.

In $\mathrm{MM} / \mathrm{NM}$ tumors, the response to NAC is not always related to the shrinking of tumor extent. However, in FM tumors, large changes in ADC indicate shrinking of the residual tumor. FM tumors most commonly decreased in size following NAC, and were centric to the initial tumor. Changes in the ADC of FM tumors may thus be correlated to the reduction rates of the tumor extent and density. In the case of FM, which could not be visualized on DWI after NAC, we believed the tumors to be pCR or near-pCR.

Changes in the delayed enhancement pattern correlated with the response rate and changes in ADC for FM but not for MM/NM. In FM, changes in ADC may reflect quantitative changes in cellularity and vascularity. In contrast, the response to NAC may be heterogeneous and complex if the initial morphological features in $\mathrm{MM} / \mathrm{NM}$ are considered.

The present results suggest that change in ADC is useful in assessing the response to NAC in FM tumors. In FM tumors, DWI may be used instead of sequential conventional MRI to assess response to NAC. This method may be both an effective and low-cost means of assessing the tumor reduction rate. Since chemotherapy regimens usually change according to the stage of cancer or expression of human epidermal growth factor, the imaging tool used to monitor the response to NAC may be selected on a morphological basis.

To the best of our knowledge, no studies exist that report on the relationship between changes in ADC and the effect of NAC based on tumor morphology. The present results indicated that change in ADC is correlated with the tumor reduction rate in FM- but not MM/NM-type tumors.

Although the results prove the usefulness of DWI for detecting the response to NAC for localized-type breast cancer, several limitations were noted. First, the study population was relatively small, with a much smaller number of $\mathrm{MM} / \mathrm{NM}$ compared to FM tumors. Our results therefore need to be confirmed in a larger clinical study. Second, we used the bi-axial diameter product as tumor size, although the threedimensional product from tumor morphology would also be acceptable. Third, only two b-factors of 0 and $1,500 \mathrm{~s} / \mathrm{mm}^{2}$ were used. The use of multiple b-values, including those higher and lower than 1,500, would have enabled a more detailed discussion to be added to our results. However, ADC by DWI using a high b-value would be less affected by perfusion (30). Thus the smaller effect of perfusion at a high b-value $(1,500 \mathrm{~s} /$ $\mathrm{mm}^{2}$ ) used needs to be considered. In addition, we measured mean ADC values, which may have underestimated the global ADC response in heterogeneous tumors. Further studies using any alternative measurements, such as minimum, are needed to support our findings.

In conclusion, changes in ADC are well correlated with the tumor reduction rate in NAC of breast cancer in FM tumors. In MM/NM tumors, it is possible that changes in ADC do not indicate a reduction in tumor extent but reflect tumor density of the residual tumor. We therefore propose DWI as a potential tool for NAC assessment based on morphological concepts.

\section{Acknowledgements}

We thank the radiological technologists of the MRI division, Kazuo Morio, Hiroaki Yasunami, Shin Yaogawa and Ichiro Morita for the technical support, and Mr. Ryo Akema and Ms. Shiho Tokuhiro for assistance with the tables and figures.

\section{References}

1. Gilles R, Guinebretiere J-M, Toussaint C, Spielman M, Rietjens M, Petit J-Y, Contesso G, Masselot J and Vael D: Locally advanced breast cancer: contrast-enhanced subtraction MR imaging of response to preoperative chemotherapy. Radiology 191: 633-638, 1994.

2. Tsuboi N, Ogawa Y, Inomata T, Yoshida D, Yoshida S, Moriki T and Kumon M: Changes in the findings of dynamic MRI by preoperative CAF chemotherapy for patients with breast cancer of stage II and III: Pathologic correlation. Oncol Rep 6: 727-732, 1998. 
3. Weatherall PT, Evans GF, Metzger GJ, Saborrian MH and Leith AM: MRI vs. histologic measurement of breast cancer following chemotherapy: comparison with X-ray mammography and palpation. J Magn Reson Imaging 13: 868-875, 2001.

4. Balu-Maestro C, Chapellier C, Bleuse A, Chanalet I, Chauvel C and Largillier R: Imaging in evaluation of response to neoadjuvant breast cancer treatment benefits of MRI. Breast Cancer Res Treat 72: 145-152, 2002.

5. Rosen EL, Blackwell KL, Bakser JA, Soo MS, Bentley RC, Yu D, Samulski TV and Dewhirst MW: Accuracy of MRI in the detection of residual breast cancer after neoadjuvant chemotherapy. AJR Am J Roentgenol 181: 1275-1282, 2003.

6. Baudu LD, Murakami J, Murayama S, Hashiguchi N, Sakai S Masuda K, Toyoshima S, Kuroki S and Ohno S: Breast lesions correlation of contrast medium enhancement patterns on MR images with histopathologic findings and tumor angiogenesis. Radiology 200: 639-649, 1996.

7. Guo Y, Cai YQ, Cai ZL, Gao YG, An NY, Ma L, Mahankali S and Gao JH: Differentiation of clinically benign and malignant breast lesions using diffusion-weighted imaging. J Magn Reson Imaging 16: 172-178, 2002.

8. Englander SA, Ulung AM, Brem R, Glickson JD and Zijl PCM Diffusion imaging of human breast. NMR Biomed 10: 348-352, 1997.

9. Kuroki Y, Nasu K, Kuroki S, Murakami K, Hayashi T, Sekiguchi R and Nawano S: Diffusion-weighted imaging of breast cancer with the sensitivity encoding technique: analysis of the apparent diffusion coefficient value. Magn Reon Med Sci 3 : $79-85,2004$

10. Woodhams R, Matsunaga K, Kan S, Hata H, Ozaki M, Iwabuchi K, Kuranami M, Watanabe M and Hayakawa K: ADC mapping of benign and malignant breast tumors. Magn Reon Med Sci 4: 35-42, 2005.

11. Woodhams R, Matsunaga $\mathrm{K}$, Iwabuchi $\mathrm{K}$, Kan S, Hata $\mathrm{H}$, Kuranami M, Watanabe M and Hayakawa K: Diffusion weighted imaging of malignant breast tumors: the usefulness of apparent diffusion coefficient (ADC) value and ADC map for the detection of malignant breast tumors and evaluation of cancer extension. J Comput Assist Tomogr 29: 644-649, 2005.

12. Rubesova E, Grell AS, De Maertelaer V, Metens T, Chao S and Land Lemort M: Quantitive diffusion imaging in breast cancer: a clinical prospective study. J Magn Reson Imaging 24: 319-324, 2006

13. Galons JP, Altbach MI, Paine-Murrieta GD, Taylor CW and Gillies RJ: Early increases in breast tumor xenograft water mobility in response to pacritaxel therapy detected by noninvasive diffusion magnetic resonance imaging. Neoplasia 1: 113-117, 1999.

14. Pickles MD, Gibbs P, Lowry M and Turnbull LW: Diffusion changes precede size reduction in neoadjuvant treatment of breast cancer. Magn Reson Imaging 24: 843-847, 2006.

15. Nakamura S, Kenjo H, Nishio T, Kaxama T and Doi O: Efficacy of 3D-MR mammography for breast conserving surgery after neoadjuvant chemotherapy. Breast Cancer 9: 15-19, 2002.

16. Murata Y, Ogawa Y, Yoshida S, Kubota K, Itoh S, Fukumoto M, Nishioka A, Moriki T, Maeda H and Tanaka Y: Utility of initial MRI for predicting extent of residual disease after neoadjuvant chemotherapy: Analysis of 70 breast cancer patients. Oncol Rep 12: 1257-1262, 2004.
17. Tozaki M, Kobayashi T, Uno S, Aiba K, Takeyama H, Shioya H, Tabei I, Toriumi Y, Suzuki M and Fukuda K: Breast-conserving surgery after chemotherapy: value of MDCT for determining tumor distribution and shrinkage pattern. AJR Am J Roentogenol 186: 431-439, 2006.

18. American College of Radiology. Breast Imaging Reporting and Data System (BI-RADS). 4th edition. American College of Radiology, Reston, VA, pp79-89, 2003.

19. Rieber A, Zeitler H, Rosenthal H, Görich J, Kreienberg R, Brambs HJ and Tomczak R: MRI of breast cancer: influence of chemotherapy on sensitivity. Br J Radiol 70: 452-458, 1997.

20. Stehling MK, Turner R and Mansfield P: Echo-planar imaging: magnetic resonance imaging in a fraction of a second. Science 254: 43-50, 1991

21. Tan KB, Thamboo TP and Rju GC: Xanthomatous pseudotumor: a usual postchemothrapy phenomenon in breast cancer. Arch Pathol Lab Med 127: 739-741, 2003.

22. Basser PJ, Pajevic S, Pierpaoli C, Dura J and Aldroubi A: In vivo fiber tractography using DT-MRI data. Magn Reson Med 44: 625-632, 2000.

23. Rovira A, Rovira-Gols A, Pedraza S, Grive E, Molina C and Alvarez-Sobin J: Diffusion-weighted MR imaging in the acute phase of transient ischemic attacks. AJNR Am J Neuroradiol 23: 77-83, 2002.

24. Yamashita Y, Tang Y and Takahashi M: Ultrafast MR imaging of the abdomen: echo plannar imaging and diffusion-weighted imaging. J Magn Reson Imaging 8: 367-374, 1998.

25. Ichikawa $T$, Haradome $H$, Hachiya $J$, Nitatori $T$ and Araki $T$ : Diffusion-weighted MR imaging with single-shot echo-plannar imaging in the upper abdomen: preliminary clinical experience in 61 patients. Abdom Imaging 24: 456-461, 1999.

26. Ries M, Joones RA, Basseau F, Moonen CT and Grenier N: Diffusion tensor MRI of the human kidney. J Magn Reson Imaging 14: 42-49, 2001.

27. Takahara T, Imai Y, Yamashita T, Yasuda S, Nasu S and van Cauteren M: Diffusion weighted whole body imaging with background body signal suppression (DWIBS): technical improvement using free breathing, STIR and high resolusion 3D display. Radiat Med 22: 275-282, 2004.

28. Nonomura Y, Yasumoto M, Yoshimura R, Haraguchi K, Ito S, Akashi $\mathrm{T}$ and Ohashi I: Relationship between bone marrow cellularity and apparent diffusion coefficient. J Magn Reon Imaging 13: 757-760, 2001.

29. Schmithorst VJ, Dardzinski BJ and Holland SK: Simultaneous correlation of ghost and geometric distortion artifacts in EPI using a multiecho reference scan. IEEE Tran Med Imaging 20: 535-539, 2001.

30. Le Bihan D, Breton E, Lallemand D, Aubin ML, Vignaud J and Laval-Jeantet $\mathrm{M}$ : Separation of diffusion and perfusion in intraoxel incoherent motion MR imaging. Radiology 168: 692-698, 1998 